\title{
¿Histeria, Simulación o Neurosis de Renta?
}

\author{
M. Prado Ordóñez Fernández
}

\begin{abstract}
a FEA Psiquiatría, Hospital General de Ciudad Real, España.

Correspondencia: María del Prado Ordoñez Fernandez, $\mathrm{C} /$ Alarcos $\mathrm{N}^{\circ} 3,1^{\circ} \mathrm{D}$, 13001, Ciudad Real, España. Correo electrónico: mpordonez@sescam.jccm.es.
\end{abstract}

Recibido el 5 de enero de 2010.

Aceptado para su publicación el 22 de enero de 2010.

\begin{abstract}
RESUMEN
Los médicos de atención primaria y los profesionales de salud mental somos testigos de un alarmante incremento de los denominados por López lbor (1966) "enfermos-problema". Para dicho autor, los "enfermos-problema" son aquéllos que ofrecen disparidad entre los síntomas subjetivos y los objetivos. Estos enfermos ¿son histéricos, son simuladores o son neuróticos de renta? El objetivo del presente artículo es volver sobre los orígenes y evolución de las neurosis con la finalidad de sensibilizarnos a una mejor comprensión de este tipo de pacientes.

Palabras clave: Histeria, Trastornos de Conversión, Simulación.
\end{abstract}

\section{ABSTRACT}

Hysteria, Simulation or compensation neurosis?

Primary care doctors and mental health professionals are witnessing an alarming increase in what López Ibor (1966) termed "problem-patients". For this author "problem-patients" are those for whom there is a disparity between objective and subjective symptoms. Are these patients, hysterical, simulators or compensation neurotics? The aim of this article is to retrace the origins and evolution of these neuroses in order to a gain a better understanding of this type of patient.

Key words. Hysteria, Conversion Disorder, Simulation.

\section{HISTERIA}

\section{Definición}

$\mathrm{H}$. Ey define la histeria como una neurosis caracterizada por la hiperexpresividad somática de afectos inconscientes. Recordemos que neurosis es toda afección psicógena en la que los síntomas son la expresión simbólica de un conflicto psíquico que encuentra sus raíces en la historia infantil del sujeto y que constituye un compromiso entre el deseo y la defensa ${ }^{1}$.

En la neurosis histérica el mecanismo de defensa es la conversión somática, que se manifiesta en el plano psicomotriz, sensorial, o vegetativo ${ }^{2}$. En el mecanismo de conversión la libido desprendida de la representación, en el proceso de represión, es transpuesta a lo corporal ${ }^{1}$.

\section{Breve recuerdo histórico}

Esta neurosis merece un breve recorrido histórico, ya que sus principales signos son conocidos desde la antigüedad y se han extendido a toda una tradición de enfermedades sine materia, que han motivado las más vivas discusiones en los médicos de todas las épocas ${ }^{2}$. Hasta que el escocés Cullen introduce, en 1976, el concepto de neurosis, la historia de las neurosis es la historia de la histeria o histerismo ${ }^{3}$. Concebía Hipócrates (460-360 a.d.c.) las manifestaciones de la histeria como debidas a cambios dimanados de la posición uterina, describiendo los síntomas como semejantes a la epilepsia ${ }^{3}$. Esta hipótesis fue importada de los médicos egipcios (Papiros de Ebers y Kahun) y se fundamenta en el mito de la migración uterina.

Platón (427-347 a.d.c.) tenía una visión de la histeria de este tipo: "en las mujeres (...) lo que se llama matriz o útero es un animal que vive en ellas con el deseo de hacer niños. Cuando permanece mucho tiempo estéril después del 
período de pubertad apenas se le puede soportar: se indigna, va errante por todo el cuerpo, bloquea los conductos del alimento, impide la respiración, causa una molestia extraordinaria y ocasiona enfermedades de todo tipo".

Galeno (131-201 d.d.c.), cuya autoridad fue prácticamente indiscutida durante los siguientes 1.500 $a^{n}{ }^{4}{ }^{4}$, sabe, por sus disecciones, que el útero no migra por el cuerpo y considera las convulsiones histéricas debidas a la acumulación en la matriz de una sustancia tóxica semejante a la materia seminal del hombre, hablando incidentalmente de la posibilidad de histeria masculina por la misma retención seminal3. Galeno piensa que la histeria es una enfermedad causada por falta de placer sexual ${ }^{4}$.

Durante las centurias sexta a decimoquinta, los conocimientos de la histeria provienen de los médicos griegos y árabes ${ }^{3}$, ya que en la Europa medieval cristiana los síntomas histéricos se atribuían al triunfo de las fuerzas del mal y las histéricas se diagnosticaban de poseídas o de brujas.

Aunque en el siglo XVII todavía no se había abandonado la idea del origen uterino de la histeria y aún se siguen quemando histéricos e histéricas en la hoguera, ciertos médicos comienzan a hablar del origen cerebral de la histeria (Le Pois 1618 y Willis $1660)^{3}$.

No podríamos hablar de la histeria sin mencionar a Thomas Sydenham (1624-1689). Sydenham describió tan certeramente los síntomas de la histeria que poco puede añadirse, incluso hoy en día, a sus observaciones. Él se dio cuenta, por primera vez, de que los síntomas histéricos pueden simular casi todas las formas de enfermedad orgánica. Para Sydenham la histeria era la gran simuladora ${ }^{4}$.

En Francia la evolución de las ideas, desde Charcot a Babinski, ilustra las vicisitudes sufridas por la noción de histeria. Charcot había estudiado la histeria por los métodos ordinarios de observación médica y llegaba a minuciosas descripciones sintomáticas que no han caducado totalmente. Babins$\mathrm{ki}$, sorprendido ante ciertas lagunas del método de observación, quiso someterlas a un control riguroso. Como genio neurológico que era, consiguió delimitar con precisión el campo de la histeria (los fenómenos "pitiáticos" que pueden ser reproducidos por la sugestión) del de la neurología lesional. A partir de Babinski, sabemos lo que no es la histeria: una enfermedad localizable, susceptible de una definición anatomo-clínica y de una descripción por acumulación de signos. Pero Babinski fracasó en su tentativa de definir la histeria: los términos de auto-sugestión y de pitiatismo no pueden tener sentido más que si se explica lo que es la sugestión o la persuasión, lo que implica el estudio concreto y analítico de la personalidad del histérico. De tal manera que, a partir de Babinsy, la histeria ha corrido el riesgo de ser considerada, fuera de toda realidad, como una simple simulación (Boisseau) ${ }^{2}$.

A la histeria, convertida en lo que no existe para la neurología, le faltaba, sin embargo, penetrar en el interior de la "realidad" que es para el psiquiatra². Esto es lo que intentó Pierre Janet en la Salpêtrière, al estudiar las relaciones de la histeria, de la hipnosis y del automatismo psicológico. En la misma época Freud tuvo la primera intuición que debía conducirle al psicoanálisis ${ }^{2}$. Partió de la idea de que los síntomas se originaban y tomaban su sentido en el inconsciente de los enfermos. Estudiando con Breuer (1895) a una enferma, a la que este último curaba periódicamente de sus síntomas por medio de una "ab-reacción" emocional (se dice aún catarsis o liberación de lo reprimido), por la evocación de sus recuerdos, concibió la idea de la represión, de su origen sexual, y de la importancia de la transferencia afectiva en la terapéutica. La histeria entraba, de este modo, en la nueva fase de estudios que han permitido captar su contenido ${ }^{2}$.

\section{Manifestaciones Clínicas}

El conjunto, por una parte muy variado, de la sintomatología histérica, que Sydenham ya designaba como "proteiforme", se puede ordenar en tres grupos2: Paroxismos o crisis neuropáticas, alteraciones de las funciones psicomotrices del sistema nervioso y trastornos viscerales o titulares ("trastornos funcionales").

Entre los primeros se encuentran los grandes ataques de histeria (casi desaparecidos en nuestra cultura actual), las crisis sincopales, la sintomatología extrapiramidal (accesos de hipo, bostezos, temblores, sacudidas musculares, movimientos coreiformes), las crisis tetaniformes, los estados crepusculares y estados segundos, el sonambulismo, las fugas histéricas, la amnesia y los ataques catalépticos ${ }^{2}$.

Entre los segundos se hallan todo tipo de parálisis y contracturas, los espasmos (hipo, vómitos, espasmos oculo-faciales), las anestesias y los trastornos sensoriales $^{2}$ (ceguera, sordera, anosmia, etc.).

Entre los terceros incluimos la imposibilidad de tragar ("el famoso bolo histérico", sentido en el cuello o en el epigastrio), los espasmos cólicos y ciertas constipaciones. Síntomas que han revelado en el análisis su naturaleza histérica. También contamos con trastornos urinarios y genitales (dispareunia), algias (todas las localizaciones y todos los tipos de dolor pueden ser sintomáticos de la histeria), crisis urticariformes, etc ${ }^{2}$. 
"El histérico habla este lenguaje de los órganos con una especial elocuencia. Vive las metáforas en vez de hablarlas y esto es lo esencial del fenómeno de conversión somática"2.

\section{Situación actual}

Desde la década de 1980, en la que la A.P.A. (American Psychiatric Association) publica su tercera edición del DSM, la histeria desaparece y se dispersa entre los trastornos somatomorfos (que incluye el trastorno conversivo), los trastornos por estrés postraumático, los trastornos disociativos y el trastorno histriónico de la personalidad, pero es importante recordar que, siempre que la ciencia o la religión han querido controlar la estructura histérica, ésta ha buscado nuevos síntomas más acordes con los tiempos.

\section{LA NEUROSIS DE RENTA}

\section{Definición}

En las neurosis de renta existen dos componentes: a) la neurosis (un trastorno mental) y b) la actitud de renta, expresión de reivindicación del sujeto y consecuencia de legislaciones que protegen y amparan las secuelas de los accidentes o de las enfermedades. La actitud de renta es la interpretación y la postura del sujeto ante una eventualidad que estima ha sido el origen de determinados síntomas y debe ser compensado ${ }^{5}$.

Las neurosis de renta se incluyen en la clasificación internacional de enfermedades de la OMS (CIE 10) dentro de la elaboración psicológica de síntomas somáticos (F68.0). Se describen como un trastorno en el que síntomas somáticos compatibles con un trastorno, enfermedad o incapacidad física, confirmados y originalmente debidos a uno de ellos, son exageradas o prolongadas debido al estado psicológico del enfermo. Así se desarrolla un síndrome comportamental de búsqueda de atención (histriónico) que a menudo incluye también quejas que no son de origen somático (y por lo general no específicas). El dolor o la incapacidad física producen malestar y a menudo preocupación, a veces justificada, por la posibilidad de padecer un dolor o incapacidad prolongados o progresivos. El trastorno puede justificarse, a ojos del que lo padece, por la insatisfacción con el resultado del tratamiento o investigaciones realizadas o la decepción con el grado de atención personal recibida en medios sanitarios. En algunos casos parece existir una clara motivación derivada de la posibilidad de obtener indemnizaciones consecutivas a accidentes o lesiones. Sin embargo, el síndrome no remite necesariamente con rapidez cuando el litigio ha sido exitoso (CIE-10).
Las neurosis de renta surgen allí donde un marco legislativo ampara con indemnización cualquier tipo de sufrimiento sobrevenido a un ser humano ${ }^{5}$.

\section{Breve recuerdo histórico}

Se ha denominado con otros epítetos: neurosis de compensación, neurosis situacional, de deseo, accidental, de billete, rentosis, rentofilia, sinistrosis, neurosis de las víctimas de un accidente, de ganancia secundaria, simulación inconsciente, historia traumática, etc. La expresión "neurosis de renta" es traducción del alemán "Rentenneurose" Dicha expresión fue puesta en circulación por la escuela alemana durante la tercera década del siglo $X X$ y fue, y sigue siendo, un modo de referirse a ciertos trastornos de tipo neurótico en relación al padecimiento de un accidente o una enfermedad ${ }^{5}$. La neurosis de renta tiene como precursoras a las neurosis traumáticas. Los primeros antecedentes se remontan al último tercio del siglo XIX. Fue Erichsen, en 1866, el primero en estudiar a fondo y de modo sistemático varios casos relativos a accidentes de ferrocarril. Años más tarde, en 1882, Page, "cirujano inglés", dio a los trastornos funcionales aparecidos tras accidente los nombres de "railway spine" y "railway brain", señalando que el estado mental de los individuos traumatizados (accidente de ferrocarril) se asemejaba al estado mental de los histéricos ${ }^{5}$.

El "railway brain" fue asimilado a un cuadro neurasténico con rasgos hipocondríacos e histerismo ${ }^{5}$. La vinculación entre traumatismo e histerismo local había sido ya descrita por Brodie en 1837, a quien se debe la descripción de la coxalgia histérica ${ }^{5}$.

En 1884, Oppenheim describió como entidad clínica autónoma la neurosis traumática, caracterizada por una combinación de fenómenos histeriformes y neurasténicos como consecuencia de accidentes de ferrocarril (el primer ferrocarril circuló en 1830) ${ }^{5}$.

Es curiosa la vinculación en el siglo XIX entre revolución industrial, capitalismo, derechos sociales y neurosis de renta.

La Seguridad Social, como tal, nace en Alemania fruto del proceso de industrialización y la lucha de los trabajadores que comienzan a organizarse en sindicatos. Durante el gobierno del Kaiser Guillermo II, impulsadas por el Canciller Otto Von Bismarck, surgen tres leyes sociales que perduran hasta hoy y representan la base del sistema de Seguridad Social: seguro contra la enfermedad (1883), seguro contra accidentes de trabajo (1884) y seguro contra la invalidez y la vejez (1889). Fue entonces cuando un gran número de sujetos reclamaron tales beneficios, no presentando a los ojos de los examinadores padecimientos que justificasen su asignación. A 
estos sujetos se les comenzó a llamar "buscadores de renta".

Charcot, en 1885, formuló el concepto de histerotraumatismo, considerando la neurosis traumática como una variedad de histeria y con un "origen funcional", a diferencia de Oppenheim que la atribuía a alteraciones moleculares del cerebro ${ }^{5}$.

A principios del siglo $X X$ el concepto de histeria de Charcot fue sometido a una severa revisión. Banbinski (1901) situó la histeria en las lindes de la simulación, cobrando forma de un tipo de "simulación inconsciente" a la que las nociones de ganancia primaria y secundaria de Freud daban un cierto sentido. La "simulación inconsciente" quedaba centrada en el campo de la neurosis, en especial la de tipo histérico ${ }^{5}$.

La idea de que en la génesis de las neurosis traumáticas desempeñaba un papel importante la condición de asegurado y el deseo de obtener una renta ya fue estimada por los médicos de finales del XIX. Strumpell (1888) consideraba que las ideas de codicia desempeñaban un papel esencial, si no principal, en la etiología de las neurosis traumáticas. Sin embargo, Gowers (1895), aunque consciente de la posible influencia de la indemnización en la génesis, informaba de casos indemnizados que no modificaban sus síntomas clínicos ${ }^{5}$.

En 1916 Bonhoeffer, en su ponencia sobre el concepto y los puntos de vista jurídicos de las neurosis traumáticas, las califica de reacciones tendenciosas del organismo, en cuyo fondo late un deseo utilitario, considerando al traumatismo como facilitador de esta reacción ${ }^{5}$.

Años más tarde, a partir de 1926, la escuela alemana propuso la expresión de neurosis de renta para referirse a aquellos cuadros clínicos a raíz de un accidente en los que primaba el deseo ilegítimo de indemnización sobre los trastornos orgánicos ${ }^{5}$. La discusión quedaba abierta a si la reacción tendenciosa era consciente o inconsciente, voluntaria o involuntaria y sobre la influencia de factores disposicionales ${ }^{5}$.

El planteamiento de la neurosis de renta desde una perspectiva psicoanalítica ayudó a una mejor comprensión y conocimiento. Los conceptos de ganancia primaria y secundaria de Freud ayudaban a su entendimiento. Las ventajas materiales ("dinero, hospitalización") y afectivas ("cuidados"), obtenidas por el paciente a raíz del trauma eran consideradas, desde el punto de vista psicoanalítico, como ganancias secundarias que ayudarían a perpetuar el trastorno ${ }^{5}$.

Freud incluye las neurosis traumáticas dentro de las neurosis actuales, aunque señala algunas di- ferencias. Así, en Más allá del principio del placer (1920) diría: "el cuadro sintomatológico de la neurosis traumática se acerca al de la histeria por su riqueza en síntomas semejantes; pero, por regla general, lo sobrepasa por sus pronunciados signos de sufrimiento subjetivo (que evocan, en ocasiones, la hipocondría, y en otras la melancolía) y por los signos de un debilitamiento y de una perturbación más generalizada de las funciones psíquicas"6.

Von Weizsaecker (1929) constituye un hito importante en la evolución histórica del concepto de neurosis de renta. Weizseacker, influido por las teorías psicoanalíticas de Freud, considerado como el padre de la medicina psicosomática, que dirigía el hospital militar de lesionados cerebrales de Breslau, pensaba que la comprensión de las neurosis de renta sólo se revelaba a la luz de la biografía del sujeto. Para el autor era la personalidad defectuosamente estructurada lo que daba significación vindicativa y conformaba la neurosis de renta.

Para Weizsaecker, la neurosis de renta era una neurosis de derecho, una enfermedad social, desencadenada por el accidente de trabajo u otro tipo de enfermedad. En la neurosis de derecho ofensa y enfermedad aparecen íntimamente vinculadas. El enfermo es víctima del padecimiento, inocente con frecuencia de las causas que lo motivaron, y cuando el médico se muestra escéptico o rechaza su trastorno lo que hace es ahondar más en la ofensa y por consiguiente reforzar la lucha por el derecho ${ }^{5}$.

Kraepelin (1865-1926) describía la neurastenia traumática como una "distimia depresiva o malhumorada, unida a una actitud quejumbrosa, a una debilidad de la voluntad y a toda suerte de manifestaciones patológicas somáticas". Los enfermos se quejan, por lo regular, de dificultades para pensar y de mala memoria. Al examen objetivo no se pueden comprobar tales alteraciones y tan sólo se manifiesta una lentitud del pensamiento. Cuando se exigen al paciente esfuerzos mentales o físicos, por ligeros que sean, los dolores aumentan, finalmente surgen palpitaciones y pulso rápido. El paciente teme, así, toda tentativa de trabajo. Aparte de los trastornos vasomotores que le ocasionan vértigo, el paciente se ve torturado por el insomnio"6.

Los autores americanos se interesan particularmente por los llamados trastornos ocupacionales ${ }^{6}$. Kaplan utiliza el término de neurosis de compensación para referirse a la tendencia a mantener aquellos síntomas que nacen en el ambiente ocupacional originariamente como una defensa contra la posibilidad de perder las ganancias secundarias derivadas de los beneficios de la incapacidad u hospitalización. La etiqueta diagnóstica refleja la neurosis subyacente en el paciente al margen de 
los factores precipitantes. Para el autor, las ganancias secundarias ocasionadas por la compensación sirven para mantener la neurosis al reforzar tendencias infantiles agresivas y/o dependientes. También apuntaba que aquellos pacientes que poseían un pasado de buen ajuste laboral tenían mejor pronóstico en psicoterapia individual o de grupo, e insistía en que era muy importante iniciar un tratamiento psicoterapéutico antes de que se produjera una regresión prolongada y una distorsión del yo ${ }^{6}$.

Es importante para nosotros, los clínicos, recordar la existencia de trastornos graves asociados a las neurosis de renta. Estos trastornos son los llamados "querulantes de pensión". En ciertas indemnizaciones, la lucha adopta un carácter activo y los afectados, no solamente se sienten emocionalmente perjudicados, sino que creen haber recibido un trato injusto por parte de los profesionales, los peritos o la justicia, defendiéndose contra ello sin escatimar ofensas y amenazas ${ }^{6}$.

En 1908, Brissaud fue el primero en describir este síndrome como un estado psicopático especial, derivado de una interpretación errónea de la Ley, y consistente en una especie de delirio razonante, fundado sobre una idea falsa de reivindicación. A este trastorno lo denominó sinistrosis. A la sintomatología querulante se sumaba un cuadro polimorfo con trastornos motores, discinesias, temblores, tics, trastornos sensitivos y psíquicos de tipo patomínico y pseudodemencial. Este cuadro está incluido en los delirios de reivindicación de Giraud, Serieux y Capgras ${ }^{6}$.

Posteriormente el término sinistrosis se asemejó en la escuela francesa al de neurosis de renta. Las neurosis de renta desaparecen con el DSM-III, al mismo tiempo que el resto de neurosis (1980), pero aún se incluye en la CIE-10 dentro del F68.0.

\section{SIMULACIÓN}

\section{Definición}

La simulación, clasificada en el DSM-IV TR dentro de los problemas adicionales que pueden ser objeto de atención clínica, se define como la producción intencionada de síntomas físicos o psicológicos desproporcionados o falsos, motivados por incentivos externos como no realizar el servicio militar, evitar un trabajo, obtener una compensación económica, escapar de una condena criminal u obtener drogas. Bajo algunas circunstancias, la simulación puede representar un comportamiento adaptativo: por ejemplo, fingir una enfermedad mientras se está cautivo del enemigo en tiempo de guerra.

Debe sospecharse simulación si existe alguna de las combinaciones presentes:
Presentación de un contexto médico-legal (por ejemplo, la persona es enviada por el fiscal a una exploración médica).

Discrepancia acusada entre el estrés o la alteración explicados por la persona y los datos objetivos de la exploración médica.

Falta de cooperación durante la valoración diagnóstica e incumplimiento del régimen de tratamiento prescrito.

Presentación de un trastorno antisocial de la personalidad.

La simulación se diferencia del trastorno de conversión y de otros trastornos somatoformos por la producción intencionada de síntomas y por los obvios incentivos externos asociados a ella. En la simulación, a diferencia de lo que ocurre en los trastornos de conversión, los síntomas no ceden por sugestión o hipnosis.

\section{Breve recuerdo histórico}

La historia de la simulación está íntimamente ligada a la historia de la histeria. Recordemos que Sydenham (1624-1689) consideraba la histeria como la gran simuladora.

Charcot (1825-1893), defensor de la histeria como enfermedad, comentaba "me veo obligado a decir y repetir que, en mi opinión, la idea de simulación se basa demasiado frecuentemente en la ignorancia del médico"8.

Moebius de Leipzig (1853-1907) consideraba que los síntomas histéricos tenían un origen psicológico ${ }^{4}$, denominando reacciones o síntomas psicogenéticos a los funcionales que surgían de la idea de estar enfermo o deber estarlo ${ }^{3}$. En la reacción psicogenética, el individuo se refugia en la enfermedad, en la patonimia clínica, con la decidida intención de alcanzar determinada finalidad ${ }^{3}$.

Se define la patonimia clínica como la producción de síntomas psíquicos y/o somáticos, obedeciendo a tendencias conscientes o inconscientes encaminadas a la consecución de un determinado propósito ${ }^{3}$.

La involuntariedad y la inconsciencia diferencian las reacciones psicogenéticas de la producción voluntaria y consciente de síntomas clínicos (la simulación), pero entre una y otra existen transiciones sutiles e indiferenciables: ¿quién puede juzgar la voluntariedad o quién la consciencia de un propósito? Entre la reacción psicogenética y la simulación existen infinitas transiciones, matices de un mismo color, tropezándose con el invencible escollo diagnóstico entre lo real y lo fingido. No puede definirse dónde comienza el refugio voluntario de la enfermedad y dónde se inicia la producción involuntaria de síntomas ${ }^{3}$. 
Esta dificultad fue resuelta por Bumke (1877-1950), sucesor de Kraepelin, en Munich, con su conocida fórmula: simulación $=$ histeria ${ }^{3}$.

En este debate ya antiguo entre patonimia psicogenética y simulación, algunos autores como Adler advirtieron que el histérico y el simulador se refugian en la enfermedad para ganar ventajas en la lucha por la vida ${ }^{3}$.

Vallejo Nájera subraya en su Tratado de psiquiatría que la tendencia a la patonimia es propia de la naturaleza humana (todos fingimos afectos y desafectos para resultar socialmente adecuados), pero subraya que esta tendencia es mucho más fuerte en personalidades histéricas, psicopáticas y en oligofrénicos $^{3,6}$.

Bleuler opinaba que los sujetos que simulaban con persistencia y habilidad una enfermedad eran auténticos enfermos mentales, y añadía: "el demostrar una simulación no indica que el sujeto explorado esté mentalmente sano y sea responsable de sus actos"6.

Por último, mencionar a T. Szasz y recordar que para el autor la simulación, si es real, no es un campo en el que debamos inmiscuirnos los médicos, ya que, en definitiva, el término simulación expresa la condena de una conducta que sólo puede ser comprendida en su contexto psico-sociológico ${ }^{6}$.

\section{Modalidades de simulación ${ }^{7}$}

I. Disimulación: es aquélla donde el enfermo oculta la patología que padece con el fin de obtener cierto beneficio personal o de convalidar futuros actos jurídicos. Surge con mayor asiduidad en pacientes internados y que desean lograr el alta médica.

II. Parasimulación: esta modalidad se da cuando el individuo representa un evento mórbido distinto al que ya padece. Sobre este punto cabe señalar que se utiliza el término "parasimulación" para diferenciarlo de la sobresimulación, entendiendo que ésta última es una variante de aquélla. Esta modalidad, de relativa relevancia médico legal, suele verse en pacientes internados y que desean obtener beneficios secundarios por medio de simular síntomas ya padecidos o vistos en terceras personas. Ejemplo de ello podemos encontrarlo en aquellos pacientes que, además de padecer su cuadro psicótico, simulan síntomas extrapiramidales con la intencionalidad de obtener algún beneficio asistencial, generalmente para que le retiren o cambien la medicación.

III. Sobresimulación: como se ha dicho, es una variante de la anterior, donde se exagera o sebreactúa la sintomatología de un evento mórbido que pa- dece o que está simulando. Es posible detectarla en la población carcelaria que desea ser trasladada al Hospital con el fin de mejorar sus condiciones de reclusión o en aquellos internos que intentan modificar su calificación penal y ser declarados inimputables en instrucción o durante el juicio oral.

V. Metasimulación: se caracteriza por la actitud psíquica voluntaria e intencional de sostener en el tiempo la sintomatología del cuadro psiquiátrico ya desaparecido. Se observa con mayor frecuencia en el Fuero Civil cuando debe determinarse el porcentaje de incapacidad.

VI. Presimulación: consiste en simular una enfermedad antes de la comisión de un acto antijurídico con el fin de poder liberarse de la responsabilidad legal que en tal sentido le cabe. Si bien no es frecuente, se puede llegar a advertir en causas penales.

\section{Criterios de Davidson para diferenciar las Neu- rosis de Renta de la Simulación ${ }^{6}$}

La incapacidad para trabajar, combinada con la conservación de la capacidad para el asueto, sugiere la posibilidad de simulación.

La facilidad para tomar medicinas, inyecciones y otros tratamientos y acudir al médico es frecuente en los neuróticos y rara en los simuladores.

Si el paciente pertenece a una clase social "responsable" (fiable) adecuada, no es probablemente un simulador.

Si el contenido del pensamiento, de los sueños o de la conversación del paciente hace referencia, con frecuencia, a detalles del accidente atemorizante es probablemente un neurótico.

El hecho de que el paciente, cuando no es observado, realice actividades para las que alega no estar capacitado inclina a pensar en la simulación.

Los simuladores, en contraste con los neuróticos, no suelen copiar síntomas que han visto en otros durante la hospitalización.

El psicodiagnóstico puede ayudar en la diferenciación.

La aceptación fácil de operaciones quirúrgicas u hospitalización psiquiátrica puede indicar que el paciente no está simulando.

Si el paciente rechaza ofrecimientos de un empleo, en el que no tendría que utilizar habilidades asociadas con sus zonas afectadas, es probablemente un simulador. 
La satisfacción evidente con nuevos exámenes, particularmente si son realizados por grupos de médicos, es más consistente con la neurosis.

\section{Consejos para el médico que se enfrenta a este tipo de "Pacientes-Problema"}

Recordar que no es responsabilidad de un clínico probar la simulación, sino ayudar al que sufre, diagnosticando y tratando de acuerdo con los estándares y procedimientos científicos, legales y profesionales aceptados. La simulación es un diagnóstico forense ${ }^{8}$.

Entrevistas cálidas y empáticas, evitando las complicaciones contratransferenciales (actitudes frías, distantes, sarcásticas, inculpatorias, confrontadoras, etc. $)^{8}$. El médico debe conocer y "controlar" la rabia impotente que invariablemente aparece cuando un paciente frustra todos sus esfuerzos diagnósticos y terapéuticos. Esta rabia impotente no solo la despiertan los simuladores, sino muchos pacientes neuróticos y algunos pacientes con personalidades narcisistas.

Ahondar en la psicobiografía del enfermo para poder comprender la elaboración psicológica de sus síntomas.

No es papel del médico castigar o disciplinar al paciente, sino comprender, ayudar y aconsejar en libertad $^{8}$.
Explicar con tacto la atipicidad del diagnóstico, en caso de que la presente, con ejemplos claros.

Derivar al paciente a psicoterapia lo antes posible para evitar el refuerzo de los síntomas y la regresión $^{8}$. La psicoterapia debe incluir la orientación a la reinserción laboral del paciente.

NOTA: Si el paciente es un simulador no tomará el tratamiento ni acudirá a psicoterapia, pero habrá aprendido que su doctor es una persona cálida, respetuosa y de fiar, a la que poder acudir cuando se encuentre verdaderamente enfermo.

\section{BIBLIOGRAFÍA}

1. Laplanche y Pontalis JB. Diccionario de psicoanálisis. BarceIona: Labor S.A.; 1983.

2. Ey $\mathrm{H}$, Bernard $\mathrm{P}$, Brisset $\mathrm{CH}$. Tratado de Psiquiatría. Barcelona: Toray-Masson S.A.; 1965.

3. Vallejo Nágera A. Tratado de Psiquiatría. Barcelona: Salvat Editores S.A.; 1944.

4. Franz G, Alexander MD, Sheldon T, Selesnick MD. Historia de la Psiquiatría. Barcelona: Espaxs; 1970.

5. Calcedo-Ordóñez A. Las Ilamadas neurosis de renta. Madrid: Real Academia Nacional de Medicina; 1975.

6. Guimon Urgartechea J. Revisión crítica del concepto de neurosis de renta. Madrid: Real Academia Nacional de Medicina; 1975.

7. Bruno AH, Cabello J. Simulación en psiquiatría forense. Cuadernos de Medicina Forense. 2002; 1(2):81-93.

8. Kaplan HI, Sadock BJ. Tratado de psiquiatría. Barcelona: Salvat Editores S.A.; 1989. 